\title{
Optically Simulating a Quantum Associative Memory
}

\author{
Dan A. Ventura \\ ventura@cs.byu.edu \\ John C. Howell \\ John A. Yeazell
}

Follow this and additional works at: https://scholarsarchive.byu.edu/facpub

Part of the Computer Sciences Commons

\section{Original Publication Citation}

John Howell, John Yeazell and Dan Ventura, "Optically Simulating a Quantum Associative Memory", Physical Review A, vol. 62, article 4233, 2.

\section{BYU ScholarsArchive Citation}

Ventura, Dan A.; Howell, John C.; and Yeazell, John A., "Optically Simulating a Quantum Associative Memory" (2000). Faculty Publications. 1110.

https://scholarsarchive.byu.edu/facpub/1110

This Peer-Reviewed Article is brought to you for free and open access by BYU ScholarsArchive. It has been accepted for inclusion in Faculty Publications by an authorized administrator of BYU ScholarsArchive. For more information, please contact ellen_amatangelo@byu.edu. 


\title{
Optically simulating a quantum associative memory
}

\author{
John C. Howell and John A. Yeazell \\ Department of Physics, The Pennsylvania State University, University Park, PA 16802 \\ Dan Ventura \\ Applied Research Laboratory, The Pennsylvania State University, University Park, PA 16802
}

(May 30, 2000)

This paper discusses the realization of a quantum associative memory using linear integrated optics. An associative memory produces a full pattern of bits when presented with only a partial pattern. Quantum computers have the potential to store large numbers of patterns and hence have the ability to far surpass any classical neural network realization of an associative memory. In this work two 3-qubit associative memories will be discussed using linear integrated optics. In addition, corrupted, invented and degenerate memories are discussed.

PACS numbers: 03.67.Lx, 42.25.Hz 


\section{INTRODUCTION}

Shor's factorization algorithm [1] initiated extensive theoretical and experimental work in quantum computing. Since Shor's work, several other quantum computing algorithms have been proposed. Grover proposed a super-classical search algorithm to find an element in an unsorted array $[2,3]$. Schack showed that a quantum computer could be used to study quantum chaos [4]. More recently, Ventura and Martinez addressed the issue of associative memories and showed that quantum computers could effectively realize these devices $[5,6]$. This article proposes a linear, integrated-optics simulation of such a quantum associative memory (QuAM).

Consider the problem of associative pattern completion - learning to produce one of the full patterns when presented with only a partial pattern. Assume a set $P$ of $m$ binary patterns of length $n$. The trivial solution is simply to store the set of patterns as a lookup table. There are two reasons why this is not always the best solution. First, it requires that a unique address be associated with each pattern. Second, the lookup table requires $m n$ bits in order to store all the patterns. It is often desirable to be able to recall the patterns in an associative fashion, thus eliminating the need for explicit addressing. That is, given a partial pattern one would like to be able to "fill in" a reasonable guess as to the rest of the pattern. This may also be considered a form of generalization as the partial pattern may never have been seen during the learning of the pattern set $P$. Further, it would of course be beneficial if a smaller representation was possible. To this end, various classical associative memory schemes have been proposed, perhaps the most well-known being the Hopfield network [7] and the bidirectional associative memory [8]. These neural approaches to the pattern completion problem allow for associative pattern recall [9], but suffer severe storage restrictions. Storing patterns of length $n$ requires a network of $n$ neurons, and the number of patterns, $m$, is then limited by $m \leq k n$, where typically $0.15 \leq k \leq 0.5$. In an effort to improve on this, a QuAM that maintains the ability to recall patterns associatively while offering a storage capacity of $\mathrm{O}\left(2^{n}\right)$ using only $n$ neurons has been proposed [6].

This QuAM is composed of 2 major components: a storage algorithm that is detailed in [5] and a recall mechanism that is based on a modified version of Grover's wellknown algorithm for quantum search $[2,3]$. It is the recall operation of the QuAM algorithm that we will focus on here. In fact, due to the nature of the proposed linear optics implementation, the initial memory storage portion of the algorithm becomes a relatively simple matter. Therefore, only a brief discussion of the memory storage will be made followed by a more thorough discussion of the memory recall.

As stated, the associative memory device will be constructed using linear integrated optics. Linear integrated optics have been shown to be a straightforward environ- ment in which to study quantum algorithms [10,11]. This is a result of the fact that single photons evolve unitarily in an absorption free linear optics environment and hence there is a one to one correlation with quantum computing [10-14]. The photon evolves as it passes through a series of passive optical devices (gates) followed by measurement. Each photon traverses through the entire circuit exploring all non-zero amplitude paths. Hence each photon that traverses through the circuit represents a realization of the experiment. An ensemble of singlephotons events will yield the probabilistic outcomes of a single photon.

There are several unique features of a linear optics quantum circuit. Each degree of freedom (a particular path in the circuit) of the single photon is labeled as an eigenstate of the Hilbert space. For example, in a 3-qubit circuit there are eight basis states. Hence, there will be eight paths, one associated with each basis state. For example in figure 1, the $|000\rangle$ through $|111\rangle$ states are each labeled with a particular path. The total contribution of the probability amplitude and phase of the photon in each path yields the wavefunction of the single photon in a spatial mode basis. The operations performed on each degree of freedom (path) yield the desired quantum circuit. Unfortunately, the apparatus grows with the Hilbert space and not with the number of bits [13] due to lack of entanglement between photons. On the other hand, there is small decoherence per logic gate in a linear optics set up. Hence, linear optics is a good environment in which to study few-bit quantum algorithms. In this paper, the various aspects of the associative memory device will be discussed in terms of the 3-path bit device shown in figure 1. After the 3-path bit discussion, a 2path 1-polarization bit scheme will be presented. The latter is slightly less intuitive but easier to implement.

An important question is how does a quantum associative memory implemented using linear optics compare with classical optical implementations of associative memories, which have been well studied [15-17]. The answer is that if we were implementing a traditional associative memory in a quantum system, there would be no benefit, as classical optics already provides good implementations. However, this is not the case. The storage and recall mechanisms for the QuAM are fundamentally different than traditional associative schemes such as Hopfield, bidirectional associative memory (BAM), etc. Since classical optical approaches are implementing these traditional schemes (indeed, they have no choice as these are the best known classical associative memory algorithms), they are limited to storage capacities of $O(k n)$, where $0.15 \leq k \leq 0.5$ (as mentioned earlier). The appeal of a quantum approach to associative memory is the ability to leverage non-classical properties of quantum systems, providing for storage capacities of up to $O\left(2^{n}\right)$.

Another potential benefit of quantum associative schemes worth mentioning is the fact that they provide a natural setting for considering higher order bit patterns. 
Traditional classical methods consider only second order correlations. Modifications to the standard classical algorithms have extended this to third and fourth order correlations [18] and higher order correlations have been shown to improve recall [19].

\section{QUANTUM ASSOCIATIVE MEMORY}

\section{A. Memory Writing and Storage}

In linear optics, generating the initial superposition of states is straightforward. For example, one can generate any superposition by using a network of beam splitters with appropriately chosen reflectance and transmittance and phase delays [12]. In this particular work, an input state consisting of eigenstates with equal amplitude is desired. Linear integrated optics can generate such equal amplitude superpositions using symmetric fiber couplers $[10,11,21]$. For example, in figure 1 , an equal amplitude superposition of the $|000\rangle,|010\rangle$ and $|111\rangle$ states is prepared for the input state. This is realized by letting a photon enter one of the ports of a $3 \times 3$ fiber coupler. By adjusting the relative phases of the three output paths and connecting the outputs to the inputs of an $8 \times 8$ fiber coupler the desired state is "written" to the memory.

For the specific example shown in figure 1, the three memories in storage are

$$
M=\left\{\begin{array}{l}
000 \\
010 \\
111
\end{array}\right\}
$$

The quantum system representing the memory is thus

$$
\left|\psi_{0}\right\rangle=\frac{1}{\sqrt{3}}(|000\rangle+|010\rangle+|111\rangle)
$$

which, in vector notation will be represented as $\left|\psi_{0}\right\rangle=$ $\frac{1}{\sqrt{3}}[1,0,1,0,0,0,0,1]$.

In this setup, the memory is only stored briefly in the various paths or fibers before entering the recall portion of the circuit. Mechanisms for storing the memory for longer times can be considered. For example, the photon amplitude for each path could be held in a conditional loop and when a switch is triggered the photon amplitudes are allowed to enter the recall device. The technical challenges of the storage portion of the device are considerable. The primary purpose of this paper is to study the recall mechanism.

\section{B. Memory Recall}

The memory recall uses a modified Grover's search algorithm. In [3] Grover showed that it was possible to search for an element in a database using almost any unitary transformation. In [11] it was shown that linear integrated optics could realize Grover's search algorithm using the discrete Fourier transform (DFT) generated by $N \times N$ symmetric fiber couplers and some prescribed phase operators realized by phase delays in the appropriate paths. The matrix elements of a DFT are given by

$$
F_{a b}=e^{2 \pi i a b / N}
$$

where $0 \leq a, b \leq N-1$. The adjoint of the DFT $F^{\dagger}$ is realized by relabeling the output paths. The relabeling is done according to a $T$ operation, where $F^{\dagger}=T F$. The $T$ operation for three path bits in matrix form is given by

$$
T=\left(\begin{array}{llllllll}
1 & 0 & 0 & 0 & 0 & 0 & 0 & 0 \\
0 & 0 & 0 & 0 & 0 & 0 & 0 & 1 \\
0 & 0 & 0 & 0 & 0 & 0 & 1 & 0 \\
0 & 0 & 0 & 0 & 0 & 1 & 0 & 0 \\
0 & 0 & 0 & 0 & 1 & 0 & 0 & 0 \\
0 & 0 & 0 & 1 & 0 & 0 & 0 & 0 \\
0 & 0 & 1 & 0 & 0 & 0 & 0 & 0 \\
0 & 1 & 0 & 0 & 0 & 0 & 0 & 0
\end{array}\right)
$$

The $T$ operation simply swaps rows of the DFT. For example, the 111 path is relabeled as the 001 path. This is shown in the second and fourth fiber coupler in figure 1.

Finally, we define the operator $I_{\phi}$ that inverts the phase of state $\phi$ and define the operator $I_{M}$ such that it inverts the phase of any state representing a valid memory. (This last operator is necessary to minimize the effects of spurious memories that develop during the recall process; see [6] for details.) The phase operators are obtained by placing a $\pi$ phase delay in the appropriate paths. Then assuming that $\left|\psi_{0}\right\rangle$ represents a 3 -qubit QuAM containing valid memories, and assuming that we want to recall the memory associated with the partial pattern $\tau$, the recall mechanism is

$$
F^{-1} I_{\overline{0}} F I_{M} F^{-1} I_{\overline{0}} F I_{\tau}|\psi\rangle
$$

Now, consider the following 3-qubit associative memory example. The linear optics realization of this device is shown in figure 1. The desired outcome is to recall the memory pattern whose first two bits are 11, thus $\tau=11$ ? where '?' represents unknown and matches either 0 or 1 .

This is done by successively applying each operator from Eq. (5). First, any patterns in the memory that match the query have their phases inverted

$$
I_{11 ?}\left|\psi_{0}\right\rangle=\left|\psi_{1}\right\rangle=\frac{1}{\sqrt{3}}[1,0,1,0,0,0,0,-1]
$$

This operation inverts the phase of any state where the first two bits are equal to 1 . This phase operator is realized in figure 1 by inverting the phase of all elements that have their first two bits high. Note that there is no need to insert a phase delay in the 110 path in the circuit described in figure 1 since there is no input to this path. 
Next, the inversion about average operation effected by the operator sequence $-F^{-1} I_{\overline{0}} F$ is performed

$$
-F^{-1} I_{\overline{0}} F\left|\psi_{1}\right\rangle=\left|\psi_{2}\right\rangle=\frac{1}{\sqrt{48}}[-3,1,-3,1,1,1,1,5]
$$

This inversion-about-average operation is realized by a balanced 8-path Mach-Zehnder interferometer consisting of two $8 \times 8$ fiber couplers. Notice the appearance of the 5 spurious memory patterns (those with amplitude $\frac{1}{\sqrt{48}}$ ). At this point, if we were to observe the system,there is a $\frac{25}{48} \approx 52 \%$ chance of recalling the appropriate pattern, $|111\rangle$. Continuing with the operator sequence of Eq. (5), the phases of all valid memory states are inverted.

$$
I_{M}\left|\psi_{2}\right\rangle=\left|\psi_{3}\right\rangle=\frac{1}{\sqrt{48}}[3,1,3,1,1,1,1,-5]
$$

This is realized by placing a phase delay in all of the paths that have a valid memory. In order to get the correct output to the correct input in figure 1, the fibers are crossed. However, there is no interaction between the fibers. Once again the rotation about average is performed yielding

$$
-F^{-1} I_{\overline{0}} F\left|\psi_{3}\right\rangle=\left|\psi_{4}\right\rangle=\frac{1}{\sqrt{192}}[-3,1,-3,1,1,1,1,13]
$$

Observing the system will yield a $\frac{169}{192} \approx 88 \%$ chance of seeing the correct pattern, $|111\rangle$. Hence, there is an $88 \%$ chance that a photon will exit the 111 port of the last $8 \times 8$ coupler. A standard voting scheme will quickly indicate that the desired state was found. Hence, introducing an ensemble of single photons to the apparatus will show roughly $88 \%$ of the photons coming out of the 111 port.

\section{CORRUPTED, INVENTED OR DEGENERATE MEMORIES}

In the last section a specific memory search was performed. There were two key elements to the search. The first element was that the memory was a valid memory. Second, the search was made for a valid element. If those two conditions were satisfied, then the search was successful $88 \%$ of the time. This three-bit search required only two iterations of the inversion about the average operator to achieve this high probability of success.

In this section, other types of memory outcomes are discussed. We classify these memories as corrupted, invented or degenerate. A corrupted memory is one in which the storage doesn't actually contain one or more of the memories that were initially intended to be in the storage. This can happen through decoherence, incorrect state preparation etc. For example, suppose that the correct memory is the one discussed earlier described by the vector $\frac{1}{\sqrt{3}}[1,0,1,0,0,0,0,1]$. However, through some process the memory has been corrupted and evolved to $\frac{1}{\sqrt{3}}[1,0,1,0,0,0,1,0]$. Then after implementing the recall, the result yields $\frac{1}{\sqrt{192}}[1,5,1,5,5,5,-3,9]$. This means there is an $\frac{81}{192} \approx 42 \%$ probability of measuring the photon in the 111 path after the search. If the corrupted memory was a result of a systematic error, such that it could be repeated, then a voting scheme (or an ensemble of experiments) would show a definitely distinct result from the "good" memory probabilities.

An invented memory is one which does not exist, but one decides to search for the memory, anyway. This is a memory pattern that was never originally intended to be in the storage. However, either by mistake or curiosity one decides to search for it. As an example, suppose that the initial state is $\frac{1}{\sqrt{2}}[1,0,1,0,0,0,0,0]$. After implementing the recall that searches for $\tau=11$ ?, the output state is $\frac{1}{\sqrt{128}}[2,2,2,2,2,2,2,10]$. Therefore there is a $\frac{100}{128} \approx 78 \%$ chance of recalling the $|111\rangle$ state that didn't initially exist. Since this is a reproducible result, it can be distinguished from the good memory after many experiments. While the probability of finding the photon in the 111 path is distinguishable from the good memory result, the result still shows a high probability of finding the invented memory. In addition, it would take a relatively large number of experiments in order to significantly (in the statistical sense) distinguish between the two. Also, as a note, the corrupted and invented memories, can, in many instances have the same statistical outcomes.

A degenerate memory is best described by "cramming" one part of the Hilbert space with many memories while leaving other parts of the Hilbert space relatively sparsely populated. This creates a rather peculiar result, if one is searching for memories in the "dense" region of the Hilbert space. It is possible in this case, when inverting about average, to actually have a negative average and hence amplify the states outside the search. Even for a perfectly good memory and valid search operation, undesirable amplifications take place. Consider an initial state vector of $\frac{1}{\sqrt{3}}[1,0,0,0,0,0,1,1]$. Hence, there are two memories associated with the query $\tau=11$ ? and only one other memory in the Hilbert space. The circuit is changed appropriately for this particular search. Once again, we search for a pattern(s) whose first two bits are 11 . The output of the recall is $\frac{1}{\sqrt{192}}[-13,-1,-1,-1,-1,-1,3,3]$. It can be seen that the valid memory outside the search is the state that is actually amplified by the search. It can be argued that a degenerate memory is of little use, since it doesn't utilize the Hilbert space well. This implies that some advanced planning should be involved in how to store the patterns so as to avoid such results. 


\section{SIMPLIFIED SETUP: 2-PATH AND 1 POLARIZATION BIT}

It is difficult to align and control 8 paths. The 3 path bit representation shown in figure 1 does provide a straightforward demonstration of the evolution of each basis state. However, a more technically feasible realization could be accomplished using a 2-path bit, 1polarization bit scheme. In this way, only 4 paths would need to be aligned, which would dramatically decrease the relative path length demands.

There are a few differences in the operators needed to implement the simpler recall mechanism. Consider figure 2. It can be seen that $4 \times 4$ couplers and polarization half wave plates (HWP) with fast axis at $22.5^{\circ}$ are used instead of the $8 \times 8$ couplers. The matrix needed for this transformation is generated by taking the tensor product of a DFT on two bits with the Hadamard transformation on one bit. The Hadamard transformation

$$
H=\frac{1}{\sqrt{2}}\left(\begin{array}{cc}
1 & 1 \\
1 & -1
\end{array}\right)
$$

is effected by placing a half wave plate (HWP) with fast axis at $22.5^{\circ}$ with respect to one of the eigenpolarizations. If one assumes that horizontally polarized light represents the $|0\rangle$, then the $22.5^{\circ}$ fast-axis orientation of the half wave plate will put the photon in an equal amplitude superposition of the two polarization states. Using this in combination with the DFT allows us to construct the inversion about average operator. A HWP at $45^{\circ}$ will rotate the polarization from one polarization state to another. This operation is later used to generate the initial superposition state. A HWP at $0^{\circ}$ will create a half wavelength phase delay for the vertical polarization. Likewise, a HWP at $90^{\circ}$ will create a half wavelength phase delay for the horizontal polarization. These last two operators are used to generate the desired phase matrices.

With the above operations, the simplified circuit can be constructed as shown in figure 2. The spatial modes are used for the first two bits and the polarization modes will be used for the third bit. In this example the horizontal polarization state will be the $|0\rangle$ state and the vertical polarization state will be the $|1\rangle$ state. A horizontally polarized photon is introduced into one of the inputs of a $3 \times 3$ coupler. The coupler generates the equal amplitude spatial mode superposition. The first $45^{\circ} \mathrm{HWP}$ rotates the horizontally polarized light in the 11 path to the vertical state. Hence the desired memory is now stored in the system.

The recall mechanism proceeds much the same way as the 3-path bit explained earlier. First, a $\pi$ phase delay swaps the phase of the states where the first two bits are high. The first two couplers and the half wave plates perform the inversion about average operation. As a note, it can be seen that a "compiling" technique similar to the one prescribed in [14] could simplify this circuit. For example, the first set of HWPs at $22.5^{\circ}$ in each path rotates the polarization to an equal amplitude superposition. Only the 00 path has an intermediate operation before performing the the inverse $-22.5^{\circ}$ operation. Hence, there is no net change to the circuit if the HWPs in the 01, 10 and 11 paths are eliminated in figure 2. The $I_{M}$ operation is performed by inserting the HWPs at the appropriate angles. For example, a valid memory is the 000 and hence a HWP in the 00 path with its fast axis rotated $90^{\circ}$ with respect to the horizontal axis will delay the $00 \mathrm{H}$ phase by $\pi$. Again, the inversion about average operation is repeated. The final state of the photon is then measured. The probabilistic results are the same as described using the 3-path bit setup.

\section{DISCUSSION}

The ideas presented in this paper have been discussed in terms of a linear optics circuit. One of the attractive features of this linear optics environment is that classical light fields which carry an ensemble of single photons can be used. Hence, the voting scheme is simply realized by having many photons propagate through the circuit. The relative intensities at the output ports are equal to the single photon probabilities. Such luxuries are not afforded by more standard quantum computing systems, which makes the idea of voting more difficult to realize. Therefore in standard setups, it would be much more difficult to distinguish between good, corrupted, invented and degenerate memories in standard systems.

The idea of the "Oracle" which Grover discusses in his original search proposal has not been mentioned so far. The primary purpose of the oracle is to "mark" a prescribed state by inverting its phase. The states with inverted phase are the states that are searched for. An excellent example of such a device for linear optics is found in [14]. In this work, the role of the oracle has been played by the person who constructs the circuit. The oracle appropriately places phase delays and waveplates in the desired paths to obtain the desired circuit. Hence, it is a less general oracle than what Grover described, but it suits the needs of the associative memory presented here.

The implementation of a many-path circuit is nontrivial. Spurious phase shifts, unequal amplitude splittings, and absorption can alter the state from the desired outcome. Several simulations were performed using nonideal elements. Consider Fig. 3. In this figure three bars show the results of simulations of the normalized relative intensity coming out of the 111 path for ideal and nonideal situations. In the simulation the initial state vector was given by $\left|\psi_{0}\right\rangle=\frac{1}{\sqrt{3}}[1,0,1,0,0,0,0,1]$. The search looked for all patterns where the first two bits were high and the third bit was unknown $(|11 ?\rangle)$. The first bar shows the relative intensity in the ideal situation (as discussed earlier). The second bar shows the normalized relative inten- 
sity when a typical $8 \times 8$ fiber coupler is introduced into the system. The amplitude splittings of an actual $8 \times 8$ fiber coupler were used in this simulation [22] (the actual $8 \times 8$ coupler was used to simulate all 4 couplers in the circuit). Notice that very little difference in normalized relative intensity can be observed. However, there is only a $62.5 \%$ probability that the photon will make it to the end of the circuit without being absorbed in the actual $8 \times 8$ fiber couplers. On the other hand, the loss was well balanced, and hence the fringe visibility remained high. The third bar shows the nonideal coupler and random spurious phase shifts in both inversion-about-average operations. For example, the spurious phase shifts used in the first inversion-about-average operation were $2 \pi / 25$, $3 \pi / 50,-\pi / 10, \pi / 100,-\pi / 50,3 \pi / 50,0,-\pi / 25$. Even with passive thermal stabilization, thermal drifts (phase shifts) can be as small as a few degrees of phase shift per minute [23]. Hence, these phase shifts are reasonable.

Lastly, consider Fig. 4. This figure shows the normalized relative output intensity coming out of the 111 path using nonideal couplers. The phases in all of the paths are ideal except for the 111 path in the first inversion-aboutaverage operation, where the phase is varied continuously from $0^{\circ}$ to $180^{\circ}$. As a note, adjusting the phases in the other paths had similar effect, which was to lower the probability of measuring a photon in the 111 path with increased phase.

In conclusion, this article has discussed a proposal for an experimental realization of a quantum associative memory. Quantum associative memories have the capability to greatly surpass current classical associative memories.

[1] P.W. Shor, in Proceeding of the 35th Symposium on Foundations of Computer Science, edited by S. Goldwasser (IEEE, Los Alamitos, CA, 1994), pp. 124-134.

[2] L.K. Grover, Phys. Rev. Lett. 79, 4709 (1997).

[3] L.K. Grover, Phys. Rev. Lett. 80, 4329 (1998).

[4] R. Schack, Phys. Rev. A. 57, 1634 (1998).

[5] D. Ventura and T. Martinez, Found. Phys. Lett., 12, 547 (1999)

[6] D. Ventura and T. Martinez, Inf. Sci., 124, 273 (2000)

[7] J.J. Hopfield , Proc. Nat. Acad. Sci., 79, 2554 (1982)

[8] B. Kosko, IEEE Trans. Sys. Man, and Cyber. 18, 49 (1988)

[9] T. Aoyagi and M. Nomura, Phys. Rev. Lett. 83, 1062 (1999)

[10] J.C. Howell and J. A. Yeazell, Phys. Rev. A 61, 012304-1 (2000)

[11] J.C. Howell and J. A. Yeazell, Phys. Rev. A 61, 052303-1 (2000)

[12] M. Reck, A. Zeilinger, H. J. Bernstein, and P Bertani, Phys. Rev. Lett. 73, 58(1994).

[13] N. J. Cerf, C. Adami, and P. G. Kwiat, Phys. Rev. A.
57, R1477(1998).

[14] P. G. Kwiat, J. R Mitchell, P. D. D. Schwindt, and A. G. White, LANL xxx-archive quant-ph9905086 (1999)

[15] S. Chang, K. Wong, W. Zhang and Y. Zhang, Appl. Opt. 38, 5032 (1999)

[16] X-M Wang, T.J. Hall and J. Wang, Appl. Opt. 34, 7565 (1995).

[17] X. Wang, G. Mu and Y. Zhang, Opt. Lett. 16, 100 (1991)

[18] C. Bak and M. Little, Proc. Intl. Conf. on Neural Networks, vol. I, pp. 181 San Diego CA, 24-27 July, IEEE Press, Piscataway NJ (1988)

[19] B.S. Kiselyov, N.Y. Kulakov, A. L. Mikaelian and V.A. Shkitin, Opt. Eng. 31, 764 (1992).

[20] R. J. C. Spreeuw, Found. Phys. 28, 361 (1998)

[21] G. Weihs, M. Reck, H. Weinfurter, and A. Zeilinger Opt. lett. 21, 302 (1996).

[22] In our simulation we used the actual amplitude splittings of a standard $8 \times 8$ fiber coupler in our lab.

[23] We built an enclosure for a fiber Mach-Zehnder interferometer (2 meter arms) in our lab and observed about 10 degrees of relative phase shift in a 1 minute time period. It is expected that this could be drastically improved with active stabilization and shorter interferometer arms.

FIG. 1. 3-path bit quantum associative memory. $\gamma$ represents a single-photon input. The small boxes in the paths are phase delays. The large boxes represent single-mode symmetric fiber couplers with the indicated dimensions.

FIG. 2. 2-path and 1-polarization bit quantum associative memory device. $\gamma_{H}$ represents a single photon of horizontal polarization at the input of the device. The small open box in the 111 path is a phase delay. The small shaded boxes in the paths are half wave plates at the specified orientation. The large boxes represent single-mode symmetric fiber couplers with the indicated dimensions.

FIG. 3. Normalized relative intensities out of 111 path. The first bar shows the normalized relative intensity coming out of the 111 path for an ideal circuit (equal amplitude splittings, no spurious phase shifts). The second bar shows the normalized relative intensity coming out of the 111 path for the nonideal situation of unequal amplitude splittings for a typical $8 \times 8$ fiber coupler. The third bar also shows the normalized relative intensity coming out of the 111 path for the nonideal situation in which unequal amplitude splittings and randomly chosen spurious phase shifts are present.

FIG. 4. Probability of measuring a photon out of the 111 path as a function of a spurious phase shift in the 111 path of the first inversion-about-average operation. 